\title{
Thermodynamically Anomalous Regions As A Mixed Phase Signal
}

\author{
K.A. Bugaev ${ }^{1, *}$, A.I. Ivanytskyi ${ }^{1}$, D.R. Oliinychenko ${ }^{1,2}$, V.V. Sagun ${ }^{1}$, \\ I.N. Mishustin ${ }^{2,3}$, D.H. Rischke ${ }^{4}$, L.M. Satarov ${ }^{2,3}$ and G.M. Zinovjev ${ }^{1}$ \\ ${ }^{1}$ Bogolyubov Institute for Theoretical Physics, of the National Academy of Sciences of Ukraine, \\ Metrologichna str. $14^{B}$, Kiev 03680, Ukraine \\ ${ }^{2}$ FIAS, Goethe-University, Ruth-Moufang Str. 1, 60438 Frankfurt upon Main, Germany \\ ${ }^{3}$ Kurchatov Institute, Russian Research Center, Akademika Kurchatova Sqr., Moscow, 123182, \\ Russia \\ ${ }^{4}$ Institute for Theoretical Physics, Goethe-University, Ruth-Moufang Str. 1, 60438 Frankfurt \\ upon Main, Germany \\ *e-mail: bugaev@th.physik.uni-frankfurt.de
}

\begin{abstract}
Using the most advanced model of the hadron resonance gas we reveal, at chemical freeze-out, remarkable irregularities such as an abrupt change of the effective number of degrees of freedom and plateaus in the collision-energy dependence of the entropy per baryon, total pion number per baryon, and thermal pion number per baryon at laboratory energies 6.9-11.6 AGeV. On the basis of the generalized shock adiabat model we show that these plateaus give evidence for the thermodynamic anomalous properties of the mixed phase at its boundary to the quark-gluon plasma (QGP). A new signal for QGP formation is suggested and justified.
\end{abstract}

PACS:25.75.Nq, 25.75.-q, 25.75.Dw

\section{INTRODUCTION}

In the last thirty years of searching for the QGP in heavy-ion collision experiments many signals of its formation were suggested, but neither direct evidence for the QGP nor a clear signal of a QGP-hadron mixed phase have been observed so far. Although some irregularities, known in the literature as the Kink [1, the Strangeness Horn [2] and the Step [3], were 


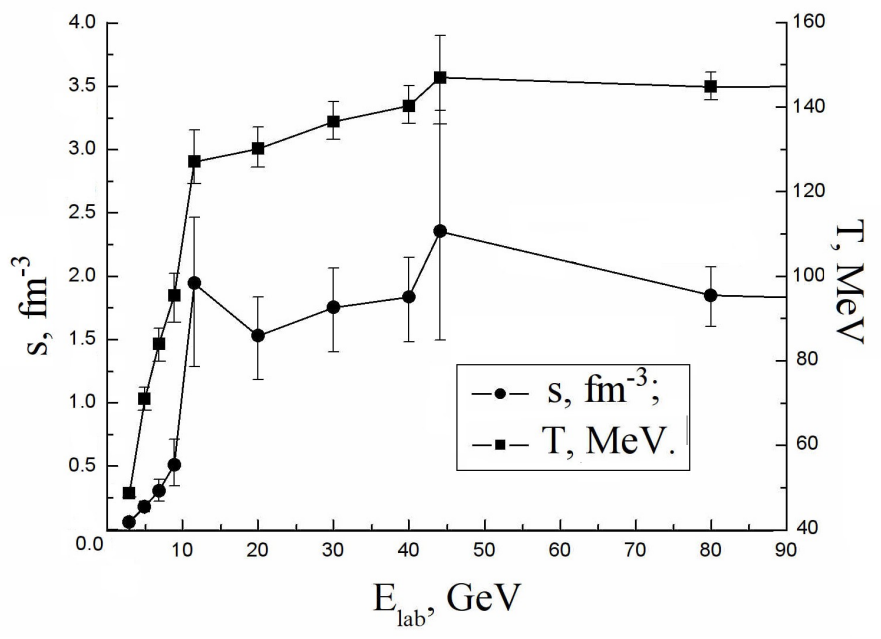

FIG. 1: Energy dependence of entropy density (circles) and temperature (squares) at chemical freeze-out extracted in [6] from hadron multiplicities measured in heavy-ion collisions.

observed and are considered to be signals of the onset of deconfinement [4], their relation to the QGP-hadron mixed phase is far from being clear. Therefore, additional and independent justification of these irregularities is required. This task is rather important in view of the planned heavy-ion collision experiments at JINR-NICA and GSI-FAIR. Evidently, searching for other irregularities and signals of mixed-phase formation and their justification is no less significant, but until recently such efforts were not very successful, since they require a realistic model which is able to accurately describe the existing experimental data and, thus, provide us with reliable information about the late stages of the heavy-ion collision process.

The recent extensions [5 8] of the hadron resonance gas model [9 13] provide us with the most successful description of available hadronic multiplicities measured in heavy-ion collisions at AGS, SPS, and RHIC energies. The global values of $\chi^{2} /$ dof $\simeq 1.16$ and $\chi^{2} /$ dof $\simeq 1.06$ achieved, respectively, in [6] and [7] for 111 independent multiplicity ratios measured at fourteen values of collision energy give us confidence that the irregularities shown in Figs. 1 and 2 are not artifacts of the model and indeed reflect reality. From Fig. 1 one can see that the entropy density $s$ increases by a factor of 4 in a range of laboratory energies per nucleon $E_{l a b} \simeq 8.9-11.6 \mathrm{GeV}$. At the same time, the chemical freeze-out (FO) temperature changes from 95 to $127 \mathrm{MeV}$ and the baryonic chemical potential $\mu_{B}$ drops from 586 to $531 \mathrm{MeV}$ [6]. In other words, for a $30 \%$ increase in the laboratory energy the 


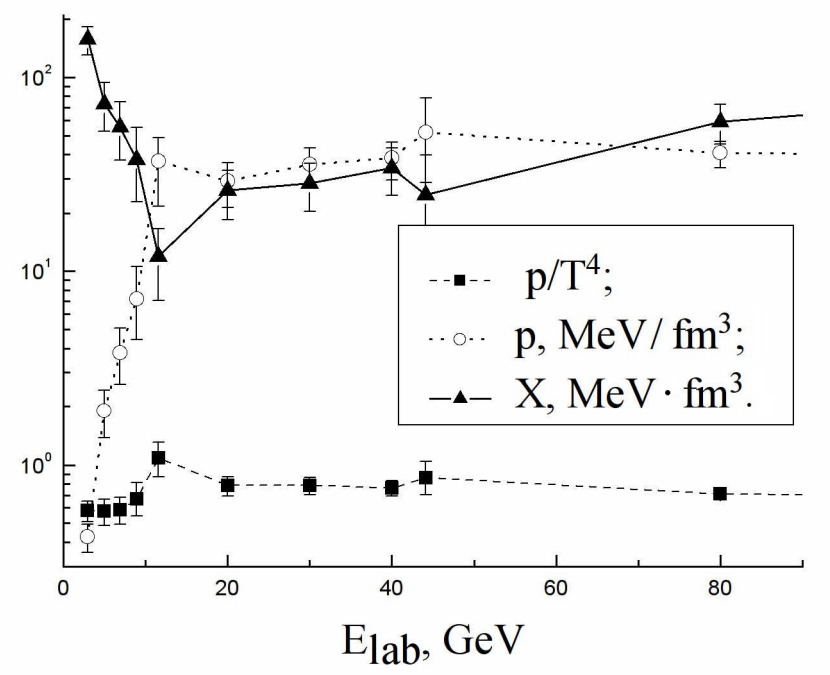

FIG. 2: Energy dependence of the chemical freeze-out pressure (circles), the effective number of degrees of freedom (squares), and the generalized specific volume $X$ found by the model of [6].

ratio $s / T^{3}$ increases by $70 \%$. A similar change can be seen in the effective number of degrees of freedom in the same energy range, cf. Fig. 2 for the chemical FO pressure in units of $T^{4}$. Note that a similar (and a somewhat stronger) rapid change in the number of effective degrees of freedom is observed in the most recent (former) version of the hadron resonance gas model [7, 8] (5, 12]).

As one can see from Fig. 2, at chemical FO a more dramatic change is experienced by the so-called generalized specific volume $X=\frac{\varepsilon+p}{\rho_{B}^{2}}$, where $\varepsilon$ is the energy density, $p$ the pressure, and $\rho_{B}$ the baryonic charge density. It is remarkable that in all known examples of equations of state (EOS) describing the QGP-hadron transition a local minimum in the energy dependence of the $X$ values of matter described by the shock or generalized shock model is observed right at the transition to the QGP, independently of whether this is a firstorder phase transition [14 17] or a strong cross-over [14, 15]. Therefore, here we would like to reanalyze the generalized shock adiabat model developed in [14-18] in order to interpret the above irregularities and to verify the other signals of mixed-phase formation. 


\section{GENERALIZED SHOCK ADIABAT MODEL}

Such a model was developed in [14 18] to extend the compression shock model [19 23] for regions of matter with anomalous thermodynamic properties. Similarly to nonrelativistic hydrodynamics [24], in the relativistic case the matter is thermodynamically normal, if

the quantity $\Sigma \equiv\left(\frac{\partial^{2} p}{\partial X^{2}}\right)_{s / \rho_{B}}^{-1}$ is positive along the Poisson adiabat. Otherwise, for $\Sigma<0$, the matter has thermodynamically anomalous properties. The sign of $\Sigma$ defines the type of allowed simple and shock waves: for $\Sigma>0$ rarefaction simple waves and compression shocks are stable. In the case of an anomalous medium compressional simple waves and rarefaction shocks are stable. If both signs of $\Sigma$ are possible, then a more detailed investigation of the possible flow patterns is necessary [15, 17]. In fact, all known pure phases have thermodynamically normal properties, whereas anomalous properties may appear at a first-order phase transition [25, 26], at its second-order critical endpoint [24], or for a fast cross-over [15].

The compression shock model of central nuclear collisions [19 23] allows one to determine the initial conditions for the subsequent hydrodynamic evolution. Such a picture of the collision process, which neglects the nuclear transparency, can be reasonably well justified at intermediate collision energies per nucleon $1 \mathrm{GeV} \leq E_{l a b} \leq 15 \mathrm{GeV}$. At laboratory energies per nucleon up to $20 \mathrm{GeV}$ this model can be used for quantitative estimates, while at higher energies it provides a qualitative description only. In the center-of-mass frame of the two colliding nuclei the initial moment of the collision can be considered as a hydrodynamic Riemann problem of an initial discontinuity. For normal media this kind of initial discontinuity leads to an appearance of two compression shocks that move in opposite directions toward the vacuum, leaving high-density matter at rest behind the shock fronts. The thermodynamic parameters $X, p, \rho_{B}$ of this compressed matter are related by the Rankine-Hugoniot-Taub (RHT) adiabat [24] with uncompressed matter in the state $\left(X_{0}, p_{0}, \rho_{B 0}\right)$,

$\rho_{B}^{2} X^{2}-\rho_{B 0}^{2} X_{0}^{2}=\left(p-p_{0}\right)\left(X+X_{0}\right)$.

This equation follows from the usual hydrodynamic conservation laws of energy, momentum, and baryonic charge across the shock front. The variable $X$ is convenient, since with its help the conserved baryonic current can be expressed as $j_{B}^{2}=-\frac{p-p_{0}}{X-X_{0}}$, i.e., in the $X-p$ plane the state existing behind the shock front is given by the intersection point of the RHT adiabat (1) and the straight line with the slope $j_{B}^{2}$ known as the Raleigh line. To solve Eq. (1) one 
needs to know the EOS. Within the compression shock model the laboratory energy per nucleon is

$E_{l a b}=2 m_{N}\left[\frac{\left(\varepsilon+p_{0}\right)\left(\varepsilon_{0}+p\right)}{(\varepsilon+p)\left(\varepsilon_{0}+p_{0}\right)}-1\right]$,

where $m_{N}$ is the mean nucleon mass. A typical example for the shock adiabat is shown in Fig. 3. As one can see from this figure the shock adiabat in the pure hadronic and QGP phases exhibits the typical (concave) behavior for a normal medium, while the mixed phase (the region $\mathrm{A}_{1} \mathrm{~B}$ ) in Fig. 3 has a convex shape which is typical for matter with anomalous properties. Until now there is no complete understanding why in a phase-transition or crossover region matter exhibits anomalous thermodynamic properties. In pure gaseous or liquid phases the interaction between the constituents at short distances is repulsive and, hence, at high densities the adiabatic compressibility of matter $-\left(\frac{\partial X}{\partial p}\right)_{s / \rho_{B}}$ usually decreases for increasing pressure, i.e., $\left(\frac{\partial^{2} p}{\partial X^{2}}\right)_{s / \rho_{B}}^{-1}=\Sigma>0$. In the mixed phase there appears another possibility to compress matter: by converting the less dense phase into the more dense one. As it was found for several EOS with a first-order phase transition between hadronic gas and QGP, the phase transformation leads to an increase of the compressibility in the mixed phase at higher pressures, i.e., to anomalous thermodynamic properties. The hadronic phase of the aforementioned EOS was described by the Walecka model [27] and by a few of its more realistic phenomenological generalizations [17, 28, 29]. The appearance of anomalous thermodynamic properties for a fast cross-over can be understood similarly, if one formally considers the cross-over states as a kind of mixed phase (but without sharp phase boundary), in which, however, none of the pure phases is able to completely dominate.

From Fig. 3 one sees that the presence of anomalous matter leads to mechanically unstable parts of the RHT adiabat (segment $A_{2} B C$ in Fig. 3) which include states in the mixed and the QGP phases. This is a model of W-kind [17, 27, 29] and its RHT adiabat in the instability region should be replaced by the generalized shock adiabat [15-17]. In the region of instability the shock wave for W-kind models has to be replaced by the following hydrodynamic solution [15]: a shock between states $\mathrm{O}$ and $\mathrm{A}_{2}$ (on the RHT adiabat shown in Fig. 3), followed by a compressional simple wave (see Fig. 4); at higher energies this solution converts into two compressional shocks and a compressional simple wave moving between them. A similar situation occurs in the case of a fast cross-over (see Figs. 3 and 4 in [15] for more details). An additional solution of two compressional shocks following one 


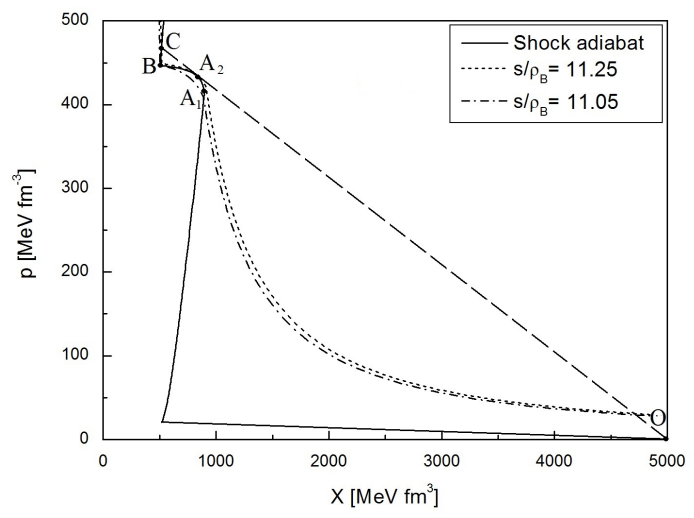

FIG. 3: The compression RHT adiabat $\mathrm{OA}_{2} \mathrm{BC}$ (solid curve) of W-kind in the $X-p$ plane. It is calculated for an EOS with first-order phase transition discussed in the text. The segments $\mathrm{OA}_{1}$, $\mathrm{A}_{1} \mathrm{~B}$, and $\mathrm{BC}$ of the adiabat correspond to the hadronic, mixed, and QGP phases, respectively. Shock transitions into the region of states $\mathrm{A}_{2} \mathrm{BC}$ are mechanically unstable. The tangent point $\mathrm{A}_{2}$ to the shock adiabat is the Chapman-Jouguet point [24]. The dotted and dashed-dotted curves show the Poisson adiabats with values of entropy per baryon specified in the legend.

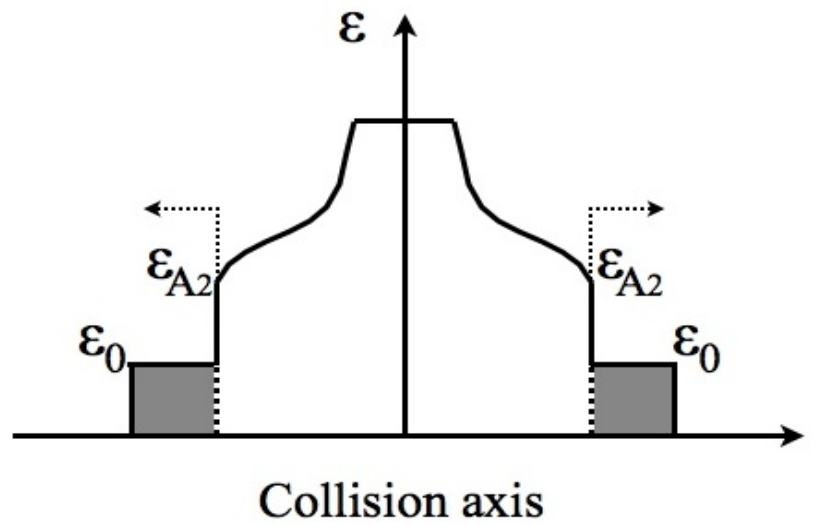

FIG. 4: Sketch of a collision of two nuclei (grey areas) where the generalized shock adiabat states are above the Chapman-Jouguet point $A_{2}$. Two shocks between the states $\varepsilon_{0} \rightarrow \varepsilon_{A_{2}}$ are followed by compressional simple waves. The dashed arrows show the direction of shock propagation.

after the other may appear, if all transitions to the mixed phase are unstable [15, 19].

Shock transitions to mechanically unstable regions are accompanied by a thermodynamic instability, i.e., the entropy in such transitions decreases, while collision energy grows [15, 25, 26. At the same time the mechanical stability condition of the generalized shock 
adiabat always leads to thermodynamic stability of its flows. Or in other words, along the correctly constructed generalized shock adiabat the entropy cannot decrease [18]. Among the possible solutions mentioned above an important role is played by the combination of a shock wave between the states $\mathrm{O}$ and $\mathrm{A}_{2}$, followed by a simple wave starting in the state $\mathrm{A}_{2}$ and continuing to states located at the boundary between the mixed phase and the QGP [15]. For such a solution the entropy is conserved, i.e., the ratio of entropy density per baryon $s / \rho_{B}=$ const, because the whole entropy production is generated by a shock $\mathrm{OA}_{2}$ to the Chapman-Jouguet point $A_{2}$ (see Fig. 3). This means that by increasing the collision energy one generates more compressed states which, however, have the same value of $s / \rho_{B}$.

Based on this solution a signal for mixed-phase formation was suggested, provided that this instability of a W-kind model exists [15-17]. The important physical consequence of such an instability is a plateau in the collison-energy dependence of the total number of pions per baryon produced in a nuclear collision, i.e., $\rho_{\pi}^{\text {tot }} / \rho_{B}\left(E_{l a b}\right) \simeq$ const [16, 17], provided by the entropy conservation during the subsequent expansion of the hydrodynamic flow formed by the generalized shock adiabat. Since the total pion multiplicity consists of thermal pions and the ones which appear from decays of hadronic resonances, in case of the RHT adiabat instability the number of thermal pions per baryon $\rho_{\pi}^{\text {th }} / \rho_{B}$ should also demonstrate a plateau or a plateau-like behavior with a small negative slope as a function of collision energy.

Note that the proposal of possible appearances of plateaus in the entropy per baryon and in the total pion number per baryon as functions of collision energy was strongly criticized in the literature. In Fig. 5 one can see all three plateaus at the laboratory energies $E_{l a b} \simeq$ 6.9 - 11.6 GeV, i.e., exactly where the other irregularities depicted in Figs. 1 and 2 occur. All quantities shown in Figs. 1, 2, and 5 were found at chemical FO within the most realistic model of the hadron resonance gas with multicomponent hard-core repulsion [6], which not only successfully describes 111 independent hadron multiplicity ratios measured for centerof-mass energies $\sqrt{s_{N N}}=2.7-200 \mathrm{GeV}$, but also correctly reproduces the energy dependence of the Strangeness Horn with a $\chi^{2} / d o f \simeq 7.5 / 14$.

It is, of course, possible that the anomalous properties of the mixed phase do not generate the mechanical instabilities for the shock transitions to this phase [15], like it was found for the Z-kind hadronic EOS [28]. Nevertheless, it was argued that even in the latter case a plateau-like structure in the entropy per baryon, and, hence, in the thermal pion multiplicity per baryon, should also be seen [15-17]. 


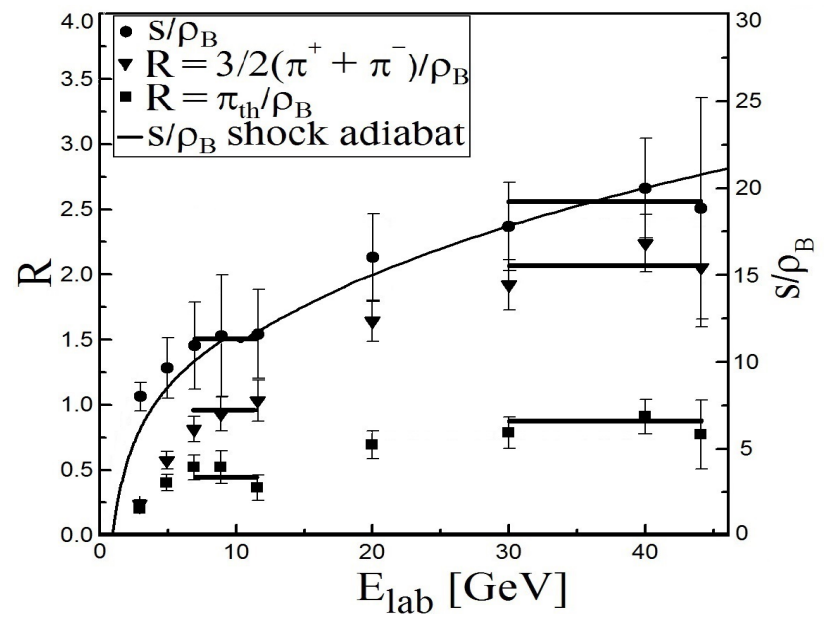

FIG. 5: Energy dependence of the entropy per baryon (circles), of the thermal pion multiplicity per baryon (squares), and of the total pion multiplicity per baryon (triangles) found at the chemical freeze-out within the realistic version of the hadron resonance gas model [6]. The horizontal bars are found by minimizing $\chi^{2} / d o f$ (see text). The solid curve corresponds to the RHT adiabat shown in Fig. 3 .

Now we are at a position to determine the parameters (individual heights for the same width) of the plateaus in the ratios $s / \rho_{B}, \rho_{\pi}^{t h} / \rho_{B}$ and $\rho_{\pi}^{t o t} / \rho_{B}$ shown in Fig. 5. We investigated a few different schemes, but came to the conclusion that a 3-parameter fit is the most reliable and simple one. Since we are searching for a plateau it is clear that its height $R_{A}$ should be the same for a given quantity $A \in\left\{s / \rho_{B} ; \rho_{\pi}^{t h} / \rho_{B} ; \rho_{\pi}^{t o t} / \rho_{B}\right\}$. The width of all plateaus in the collision energy should also be the same, since they are generated by the same physical mechanism. Let $i_{0}$ denote the beginning of plateau, while $M$ denotes its width. Then one has to minimize

$\chi^{2} / d o f=\frac{1}{3 M-3} \sum_{A} \sum_{i=i_{0}}^{i_{0}+M-1}\left(\frac{R_{A}-A_{i}}{\delta A_{i}}\right)^{2}$

for all possible values of $i_{0}$ and $M>1$. Here the subscript $i$ counts the data points $A_{i}$ to be described, whereas $\delta A_{i}$ denotes the error of the corresponding quantity $A_{i}$. We assume that the plateaus are correlated to each other, if $\chi^{2} / d o f$ is essentially smaller than 1 . Also from the practical point of view it is necessary to find the set of maximally correlated plateaus for future experiments. The height of each plateau $R_{A}$ is found by minimizing $\chi^{2} / \operatorname{dof}$ in 
(3) with respect to $R_{A}$ and one gets

$R_{A}=\sum_{i=i_{0}}^{i_{0}+M-1} \frac{A_{i}}{\left(\delta A_{i}\right)^{2}} / \sum_{i=i_{0}}^{i_{0}+M-1} \frac{1}{\left(\delta A_{i}\right)^{2}}$.

As one can see from the table below minimal values of $\chi^{2} / d o f<0.2$ are reached for $M=2$, but these are not the widest plateaus. There exist two sets for $M=3$ with $\chi^{2} / d o f \simeq 0.53$ for the low-energy plateaus (at $E_{l a b} \simeq 6.9-11.6 \mathrm{GeV}$ ) and with $\chi^{2} / d o f \simeq 0.34$ for the high-energy plateaus (at $E_{l a b} \simeq 30-40 \mathrm{GeV}$ ). Precisely these sets are depicted in Fig. 5 . since we believe that the high-energy set of $E_{l a b} \simeq 20-40 \mathrm{GeV}$ with the width $M=4$ should not be taken into account because its value of $\chi^{2} / d o f \simeq 0.87$ is too close to 1 and, therefore, such plateaus are not strongly correlated even for these huge error bars.

TABLE I: Results of the 3-parameter fit.

\begin{tabular}{|c|c|c|c|c|c|}
\hline & \multicolumn{5}{|c|}{ Low energy minimum } \\
\hline$M$ & $i_{0}$ & $R_{s / \rho_{B}}$ & $R_{\rho_{\pi}^{t h} / \rho_{B}}$ & $R_{\rho_{\pi}^{t o t} / \rho_{B}}$ & $\chi^{2} /$ dof \\
\hline 2 & 3 & 11.12988 & 0.52037 & 0.85683 & 0.17811 \\
\hline 3 & 3 & 11.31482 & 0.46128 & 0.89174 & 0.53144 \\
\hline 4 & 2 & 10.55597 & 0.43340 & 0.72523 & 1.64913 \\
\hline 5 & 2 & 11.53637 & 0.47009 & 0.84800 & 4.45466 \\
\hline & \multicolumn{5}{|c|}{ High energy minimum } \\
\hline 2 & 8 & 19.80518 & 0.88229 & 2.20373 & 0.12751 \\
\hline 3 & 7 & 18.77659 & 0.83508 & 2.05780 & 0.34045 \\
\hline 4 & 6 & 17.82325 & 0.77920 & 1.87732 & 0.87105 \\
\hline 5 & 5 & 16.26105 & 0.64800 & 1.62094 & 3.72057 \\
\hline
\end{tabular}

New signal of QGP formation.- Here we suggest a new signal indicating a boundary between the mixed phase and the QGP, which also signifies the existence of mechanical instabilities inside the mixed phase. This is an appearance of a local minimum of the generalized specific volume $X$ at chemical FO as a function of the collision energy (see Fig. 2). Note that all stable RHT adiabats of Z-kind and all unstable RHT adiabats of W-kind and the corresponding generalized shock adiabats with the QGP EOS of the MIT-Bag model type [30] studied in [15-17, 29] demonstrate exactly the same behavior. The physical origin for such a behavior is that for an increase in collision energy the entropy per baryon and 
the temperature of the formed QGP (being a normal medium) increase as well, while the baryonic density and the baryonic chemical potential are steadily decreasing. Hence in the QGP phase the variable $X \equiv\left(T s / \rho_{B}+\mu_{B}\right) / \rho_{B}$ grows, if the collision energy increases. Intuitively, such a dependence seems to be true for other QGP EOS, if they correspond to a normal medium. On the other hand, the behavior of the variable $X$ inside the mixed phase with anomalous properties is opposite and it does not depend on the stability or instability of the shock transitions to this region [15-17]. On the basis of these arguments one can understand the reason why the boundary of the mixed phase and QGP corresponds to a local minimum of the $X$ variable along the RHT (shown in Fig. 3) or generalized shock adiabat and why it is also a minimum of $X$ as function of collision energy [15 17, 29].

In case of unstable shock transitions to the mixed phase, the unstable part of the mixed phase (segment $\mathrm{A}_{2} \mathrm{~B}$ in Fig. 3) should be replaced by the Poisson adiabat passing through the point $\mathrm{A}_{2}$ (the dotted curve shown in Fig. 3). Consequently, if the matter formed in a collision expands isentropically after the shock $\mathrm{OA}_{2}$ disappears, then it can be shown that for the chemical FO pattern depicted in Figs. 1 and 2 the minimum of the variable $X$ of the initial state corresponds to a minimum of this variable at chemical FO, i.e., $\min \left\{X\left(E_{l a b}\right)\right\}$ corresponds to $\min \left\{X^{F O}\left(E_{l a b}\right)\right\}$. Indeed, the final states of the isentropic expansion belong to the Poisson adiabat at which $s / \rho_{B}=s^{F O} V^{F O} /(2 A)=$ const. Here the entropy density $s^{F O}$ and the system volume $V^{F O}$ are taken at chemical FO, while the total number of baryons in an $\mathrm{A}+\mathrm{A}$ collision is $2 \mathrm{~A}$. At chemical FO temperatures $T$ below $150 \mathrm{MeV}$, the hadronic EOS can be safely represented as a mixture of ideal gases of massive pions and nucleons, i.e., its pressure $p \simeq T\left(\rho_{B}+\rho_{\pi}\right)$ and energy density $\varepsilon \simeq\left(m_{N}+3 / 2 T\right) \rho_{B}+\left(m_{\pi}+3 / 2 T\right) \rho_{\pi}$ can be represented via the density of nucleons $\rho_{B}$ and density of pions $\rho_{\pi}$ (here $m_{N}\left(m_{\pi}\right)$ is the nucleon (pion) mass). With the help of this EOS the variable $X$ at chemical FO can be cast as $X^{F O} \simeq\left[m_{N}+m_{\pi}+5 / 2 T\left(1+\rho_{\pi} / \rho_{B}\right)\right] V^{F O} /(2 A)$. From Fig. 5 one sees that constant values of $s / \rho_{B}$ in the range of $E_{l a b}=6.9-11.7 \mathrm{GeV}$ correspond to a nearly constant ratio $\rho_{\pi} / \rho_{B} \simeq 0.5$ and, hence, one can write $X^{F O} \simeq\left[m_{N}+m_{\pi}+3.75 T\right] V^{F O} /(2 A)$ for these energies. Since for these energies the entropy density changes from $0.3 \mathrm{fm}^{-3}$ to $1.944 \mathrm{fm}^{-3}$, while the chemical FO temperature changes from $84 \mathrm{MeV}$ to $127 \mathrm{MeV}$, it is clear that approximately one can write $X^{F O} \simeq\left[m_{N}+m_{\pi}+358 \mathrm{MeV}\right] V^{F O} /(2 A)$ and, hence, the value $X^{F O} s^{F O} \simeq[1536 \mathrm{MeV}] V^{F O} s^{F O} /(2 A)=$ const. A direct numerical check shows that for the chemical FO data belonging to the laboratory energy range $E_{l a b}=6.9-11.7 \mathrm{GeV}$ one 
obtains $X^{F O} s^{F O} \simeq 16.9 ; 19.3 ; 23.1$, which means that such a relation is valid with the relative deviations $-12 \%$ and $+21 \%$. Note that the relation $X^{F O}{ }_{s}{ }^{F O} /\left[m_{N}+m_{\pi}+3.75 T\right] \simeq$ const gives us the values $15 ; 16.5 ; 17.9$, i.e., it is fulfilled with relative errors $-9.1 \%$ and $+8.5 \%$ in this energy range and these estimates validate our EOS usage. Using these arguments, we conclude that with reasonable accuracy one can establish the relation $X^{F O} \sim V^{F O} \sim 1 / s^{F O}$ for the final states which belong to the Poisson adiabat and, therefore, the growth of entropy density (see Fig. 1) and the decrease of the variable $X$ shown in Fig. 2 are directly related to each other.

The same treatment can be applied to higher energies. In this case one has to write $X^{F O} \simeq$ $\left[m_{N}+m_{\pi}+5 / 2 T\left(1+\rho_{\pi} / \rho_{B}\right)\right] s^{F O} / \rho_{B} / s^{F O}$ and account for the fact that the ratios $s^{F O} / \rho_{B}$ and $\rho_{\pi} / \rho_{B}$ are increasing with the collision energy, while the chemical FO temperature and entropy density are almost constant for $E_{l a b}>11.7 \mathrm{GeV}$ (see Fig. 1). Hence, in this case one can write $X^{F O} \simeq s^{F O} / \rho_{B}$, i.e., for laboratory energies above $11.7 \mathrm{GeV}$ the variable $X^{F O}$ should increase with the collision energy, and this is a reflection of the growth of the initial values of the $X$ variable, when the generalized shock adiabat goes inside the QGP.

The same conclusion can be obtained from the fact that the Poisson adiabats with the different $s / \rho_{B}$ values cannot intersect each other. Therefore, the generalized shock adiabat which must replace the unstable RHT adiabat (like the one shown in Fig. 3) would generate the field of nonintersecting Poisson adiabats in the $X-p$ plane, since along the mechanically stable hydrodynamic solutions the entropy cannot decrease. By construction at given $X$ the Poisson adiabat with higher value of $s / \rho_{B}$ has higher pressure $p$. Then applying the chemical FO criterion $p=$ const, which within the error bars is clearly seen in Fig. 2, to such a field of nonintersecting Poisson adiabats in the $X-p$ plane, one observes that the higher values of $s / \rho_{B}$ correspond to larger values of the variable $X^{F O}$ along the line $p=$ const.

Accounting for the above estimates, we conclude that the local minimum of the $X^{F O}$ variable is related to the minimum of the variable $X$ on the generalized shock adiabat existing at the boundary between the mixed phase and QGP. Moreover, the above estimates show that the minimum of the $X^{F O}\left(E_{l a b}\right)$ function corresponds to the minimum of the chemical freeze-out volume $V^{F O}\left(E_{l a b}\right)$, reported in [12] and reanalyzed recently in [5]. Thus, we find that the minimum of $V^{F O}\left(E_{l a b}\right)$ is generated by the unstable part of the RHT adiabat to the boundary of mixed and QGP phases, i.e., it is another signal of QGP formation.

Note that these conclusions were also verified numerically for the shock adiabat shown in 
Figs. 3 and 5 and, hence, it is appropriate to present here the employed EOS. The hadron gas pressure used in the present work accounts for the mesonic and the (anti)baryonic states which are described by the masses $m_{M}, m_{B}$ and by the temperature-dependent numbers of degrees of freedom 31

$p_{H}=\left[C_{B} T^{A_{B}} e^{-\frac{m_{B}}{T}} \cdot 2 \cosh (\mu / T)+C_{M} T^{A_{M}} e^{-\frac{m_{M}}{T}}\right] \cdot e^{-\frac{p_{H} V_{H}}{T}}$.

This EOS accounts for the short-range repulsion introduced via the excluded volume $V_{H}=$ $\frac{4}{3} \pi R_{H}^{3}$ (with $R_{H}=0.3 \mathrm{fm}$ ) taken to be equal for all hadrons. With the parameters $m_{M}=8$ $\mathrm{MeV}, m_{B}=800.5 \mathrm{MeV}$ and

$$
\begin{array}{ll}
A_{M}=4.95 & C_{M}=6.90 \cdot 10^{-9} \mathrm{MeV}^{1-A_{M}} \mathrm{fm}^{-3} \\
A_{B}=6.087, & C_{B}=2.564 \cdot 10^{-9} \mathrm{MeV}^{1-A_{B}} \mathrm{fm}^{-3}
\end{array}
$$

such a model not only represents the mass-integrated spectrum of all hadrons, but also it rather accurately reproduces the chemical FO densities of mesons $\rho_{M}$ and baryons $\rho_{B}$ and the ratios $s / \rho_{B}$ and $s / \rho_{M}$ for the chemical FO temperatures below $155 \mathrm{MeV}$ [31]. The parameters of the center of the shock adiabat were fixed as: $p_{0}=0, \rho_{0}=0.159 \mathrm{fm}^{-3}$ and $\varepsilon_{0}=126.5 \mathrm{MeV} \mathrm{fm}^{-3}$.

The QGP EOS is motivated by the MIT-Bag model [30] $p_{Q}=A_{0} T^{4}+A_{2} T^{2} \mu^{2}+A_{4} \mu^{4}-B$, where the constants $A_{0} \simeq 2.53 \cdot 10^{-5} \mathrm{MeV}^{-3} \mathrm{fm}^{-3}, A_{2} \simeq 1.51 \cdot 10^{-6} \mathrm{MeV}^{-3} \mathrm{fm}^{-3}, A_{4} \simeq$ $1.001 \cdot 10^{-9} \mathrm{MeV}^{-3} \mathrm{fm}^{-3}$, and $B \simeq 9488 \mathrm{MeV} \mathrm{fm}^{-3}$ were found by fitting the $s / \rho_{B}$ chemical FO data for $E_{l a b}<50 \mathrm{GeV}$ with $s / \rho_{B}$ values along the RHT adiabat and by keeping the pseudocritical temperature value at zero baryonic density close to $150 \mathrm{MeV}$ which is known from lattice QCD [32]. The phase diagram was found from the Gibbs criterion, $p_{H}\left(T, \mu_{B}\right)=p_{Q}\left(T, \mu_{B}\right)$. The resulting RHT adiabat describes the $s / \rho_{B}$ chemical FO data well (see Fig. 5).

\section{DISCUSSION OF THE RESULTS}

We have presented remarkable irregularities at chemical FO elucidated via a high-quality fit of experimental particle ratios obtained by the advanced version of the hadron resonance gas model. The achieved value of fit quality $\chi^{2} / d o f \simeq 1.16$ gives us a high confidence in our findings. Among these irregularities we observed a dramatic jump of the effective number 
of degrees of freedom and a local minimum of the generalized specific volume $X^{F O}\left(\sqrt{s_{N N}}\right)$ at center-of-mass collision energies $\sqrt{s_{N N}}=4.3-4.9 \mathrm{GeV}$. Also, at chemical FO we found plateaus in the collision-energy dependence of the entropy per baryon, of the total and of the thermal numbers of pions per baryon, which were predicted long ago [15 17]. We discussed the generalized shock adiabat model for low energy collisions and argued that the found plateaus and the minimum of $X^{F O}\left(\sqrt{s_{N N}}\right)$ are generated by the RHT adiabat instabilities existing at the boundary between the mixed phase and QGP. The numerical simulations of the RHT adiabat for the realistic EOS of the hadronic phase allowed us to reproduce the $s / \rho_{B}$ plateau and to fix the parameters of the QGP EOS. Also, at chemical FO we found a second set of plateaus at $E_{l a b} \simeq 30-40 \mathrm{GeV}$, which, however, do not correspond to a phase transition or to the discussed instabilities. To make more definite conclusions about the found plateaus at laboratory energy $30-40 \mathrm{GeV}$ we need more precise data measured with $E_{l a b}$ steps of about 100-200 MeV.

\section{Acknowledgments}

The authors are thankful to I. P. Yakimenko for valuable comments. This publication is based on the research provided by the grant support of the State Fund for Fundamental Research (project N $\Phi 58 / 175-2014$ ). K.A.B. and I.N.M. acknowledge a partial support provided by the Helmholtz International Center for FAIR within the framework of the LOEWE program launched by the State of Hesse. D.R.O. acknowledges funding of a Helmholtz Young Investigator Group VH-NG-822 from the Helmholtz Association and GSI, and thanks HGSHIRe for a support. A partial support by the grant NSH-932.2014.2 is acknowledged by I.N.M. and L.M.S.

[1] Gazdzicki M., Z. Phys. C 66 (1995) 659.

[2] Gazdzicki M. and Gorenstein M. I., Acta Phys. Polon. B 30 (1999) 2705.

[3] Gorenstein M. I., Gazdzicki M.and Bugaev K. A., Phys. Lett. B 567 (2003) 175.

[4] Gazdzicki M., Gorenstein M. I.and Seyboth P., Acta Phys. Polon. B 42 (2011) 307.

[5] Oliinychenko D. R., Bugaev K. A.and Sorin A. S., Ukr. J. Phys., 58 (2013) 211. 
[6] Bugaev K. A., Oliinychenko D. R., Sorin A. S. and Zinovjev G. M., Eur. Phys. J. A 49 (2013) 30.

[7] Bugaev K. A et al., Europhys. Lett., 104 (2013 22002.

[8] Bugaev K. A. et. al., arXiv: 1312.4367 (2013).

[9] Cleymans J.and Satz H., Zeit. Phys. C 57 (1993) 135.

[10] Cleymans J.and Redlich K., Phys. Rev. Lett., 81 (1998) 5284.

[11] Braun-Munzinger P., Cleymans J., Oeschler H. and Redlich K., Nucl. Phys. A 697 (2002) 902.

[12] Andronic A., Braun-Munzinger P.and Stachel J., Nucl. Phys. A 772 (2006) 167 and references therein.

[13] Andronic A., Braun-Munzinger P.and Stachel J., Phys. Lett. B 673 (2009) 142.

[14] Bugaev K. A.and Gorenstein M. I., Z. Phys. C 43 (1989) 261 and references therein.

[15] Bugaev K. A., Gorenstein M. I., Kämpfer B.and Zhdanov V. I., Phys. Rev. D 40 (1989) 2903.

[16] Bugaev K. A., Gorenstein M. I. and Rischke D.H., JETP Lett. (Russ.) 52 (1990) 1121.

[17] Bugaev K. A., Gorenstein M. I. and Rischke D. H., Phys. Lett. B 255 (1991) 18 and references therein.

[18] Bugaev K. A., Gorenstein M. I. and Zhdanov V. I., Teor. Mat. Fizika (in Russian) 80 (1989) 138.

[19] Galitskij V. M. and Mishustin I. N., Phys. Lett. B 72 (1978) 285.

[20] Stöcker H., Graebner G., Maruhn J. A. and Greiner W., Phys. Lett. B 95 (1980) 192.

[21] Kämpfer B., J. Phys. G 9 (1983) 1487.

[22] Stöcker H. and Greiner W., Phys. Rep., 137 (1986) 277 and references therein.

[23] Barz H. W., Csernai L. P., Kämpfer B.and Lukacs B., Phys. Rev. D 32 (1985) 115.

[24] Landau L. D. and Lifshitz E. M., Fluid Mechanics (Pergamon, New York, 1979).

[25] Zel'dovich Y. B. and Raiser Y. P., Physics of shock waves and high temperature hydrodynamic phenomena (Academic, New York, 1967).

[26] Rozhdestvensky B. L. and Yanenko N. N., Systems of Quasi-Linear Equations (Nauka, Moscow, 1978).

[27] Walecka J. D., Ann. Phys., 83 (1974) 491.

[28] Zimanyi J. et al., Nucl. Phys. A 484 (1988) 647.

[29] Merdeev A.V., Satarov L.M.and Mishustin I.N., Phys. Rev. C 84 (2011) 014907. 
[30] Chodos A. et. al., Phys. Rev. D 9 (1974) 3471.

[31] Bugaev K.A. et al., PoS Baldin ISHEPP XXI 12 (2012) 017.

[32] Aoki Y. et al., Phys. Lett. B 643 (2006) 46. 